\title{
Application of Virtual Cloud Technology on Computer Laboratory Construction Management
}

\author{
Lili Liang ${ }^{1, a}$, Nan $\mathrm{Gao}^{1, \mathrm{~b}}$ \\ ${ }^{1}$ Xianyang Normal University, Xianyang, Shaanxi, 712000 \\ ${ }^{\mathrm{a}}$ email, ${ }^{\mathrm{b}}$ email
}

Keywords: Virtual Cloud, Computer Laboratory, Construction Management

\begin{abstract}
The construction of computer labs based on cloud technology can lead to the formation of dynamic and diverse laboratory environments, through the cloud client resource access, making the computer laboratory construction and management methods have undergone a fundamental change, so the cost of computer lab, Resource sharing rate and other issues will be effectively improved.
\end{abstract}

\section{Introduction}

Cloud computing technology is a kind of Internet through the Internet to obtain the necessary resources and data, and can save the data on the Internet or server, greatly enhance the computer and network technology application efficiency, and this data can also get better Sharing, to achieve a simple automatic data acquisition and release. The calculation system is mainly composed of three levels of resources, platform and application. Virtual cloud technology is mainly refers to the cloud computing technology to create virtualization, you can provide a similar collection of resources for the collection interface, and thus achieve the difference between the traditional mode of operation. This technology allows the visitor to use the common method to view, maintain or modify the stored resources, which mainly includes the server, storage resources, applications, platforms and other virtualization technology to achieve the efficient integration of computer storage resources and the use of the purpose.

\section{Problems in the Management of Computer Laboratory in Chinese Universities at Present}

China's computer technology development is relatively backward, is still in the rising stage, and the major colleges and universities in the construction of computer laboratories, the management approach is relatively traditional, resulting in a variety of problems. First, the laboratory server utilization is low, even if the installation of two servers may not be able to all be applied, the main performance of one of the servers for a long time in full load operation, while the other server utilization is Qiao\% The following, resulting in higher equipment maintenance costs. Second, the current computer software and hardware updates faster, and colleges and universities computer laboratory equipment and hardware can not be updated in time, when connected to the network is likely to cause crashes, data loss or system crashes, etc., can not guarantee Running stability. Third, the computer laboratory management model is more dispersed, the traditional room occupies a larger area, in order to reasonable arrangements may use multiple rooms to store, increase the management staff workload, an increase of maintenance costs. Fourth, the laboratory data sharing rate is low, each laboratory only install the professional software and the corresponding operating system, resulting in the professional students can only enter the professional classroom to learn, greatly wasted the educational resources, data sharing almost none.

Desktop virtualization is the past, the computer operating system and software are installed in the virtual background server, all operations are carried out by the service, and teachers and students can at any time through the client's virtual account to access and operation. And the server itself can also generate virtual desktops through virtualization technology using the remote left protocol to the virtual desktop sent to the terminal. Virtual network administrator can be through the desktop IE browser to achieve access to the server, and thus achieve a centralized management of the desktop 
environment. In order to further optimize the management model, but also by the laboratory administrator to create the appropriate desktop module for students to provide personalized settings, so that the virtual account will not be restored by the computer can be recorded on the server, which You can log in to your database through a virtual account, regardless of which lab you are attending in your lab, to meet the needs of computer professional students in professional design and graduation design. In addition, the advantage of this virtual desktop is that if the virtual server is disconnected, students can continue to operate through the local system, to be reconnected after the subsequent data can be stored in the virtual server.

Virtualization of the server is more complex and requires complete device support, including software and hardware, which can improve the efficiency of the original server, reducing the peak overload. The establishment of the virtual server mainly includes the calculation of the virtual node and the choice of the control node. The virtual node is mainly based on the virtual technology to be placed on the basis of the physical server, making a physical server into multiple virtual servers, and the virtual server is also relatively independent, through real-time monitoring, data statistics, online migration and load Balance and other technologies to improve the efficiency of the use of the server.

\section{The Application of Virtual Cloud Technology on Computer Laboratory Construction Management}

Based on the cloud computing architecture, the use of virtual technology to redesign the laboratory network, the formation of virtual cloud computing laboratory platform. The virtualization of hardware, application software, system management, operating system and configuration tool is realized through Vmware Vsphere software. Virtualization of physical hardware resources CPU, network adapter, hard disk and memory is carried out through Vmware Vsphere, and finally virtual Resource pool. For better operation, the virtualization administrator needs to develop the corresponding virtual desktop template according to the experimental project. The View Connection Server management function can provide the corresponding virtual desktop templates to the various laboratories, and generate the virtual system VM and the corresponding login in batches according to the template Account, password, thus the personalized experimental environment to be formed.

Students in the lab can access the virtual system of the remote virtual lab by using the login account in the thin client, notebook and other devices where the cloud client is installed, and then operate the experiment. For students outside the lab that already have login privileges, the virtual lab resources can be accessed at random by the cloud client, so that the lab resources are shared. All users can use the cloud client to access lab resources in the lab, so the utilization of the lab is effectively enhanced.

In the traditional laboratory, the PC is scattered, and the administrator needs to install the operating system of each room. Therefore, it takes more time. The student's location is scattered, but the environment and data of the desktop can not be transferred effectively. inconvenient. Thin client and non-thin client is an effective form of virtual desktop technology, which in the PC standard based on the small industry-specific commercial PC is a thin client, there is no hard disk drive, the central server to store the data effectively. Rather than the thin client is the protection card management upgrade, in the non-management mode, the local implementation of the user terminal operation. In the virtual technology support, the virtual machine in the effective installation of the traditional PC terminal operating system, the server for all operations, the user can use the client to the virtual machine for effective access. With virtual technology, virtual desktops will appear under the cloud server, as long as the remote desktop display protocol, you can effectively distributed to the desktop on the network. Through the service management platform, the administrator can effectively manage the virtual desktop, making the desktop environment centralized management, hardware resources, such as the flexibility to allocate. Desktop virtualization, the laboratory managers only in accordance with the actual needs to create a desktop module, and as a basis for effective settings can generate a lot of virtual desktop, and finally in accordance with the user name to its rapid distribution, so management room management efficiency Will greatly enhance. With 
the support of the virtual personal desktop set up, the virtual machine data is not restored, regardless of when the user can use the device to log on to their own desktop, in order to achieve a variety of on demand. By setting the virtual teaching desktop custom restore, while setting the batch to modify the virtual machine IP, system login name, etc., in class, training, computer grade test can be effectively used. Because virtual desktops are used for security, virtual desktops can be shared with local systems, and if the network is interrupted, the user can continue with the support of the local system to complete the operation. Even if the desktop virtual, the traditional PC can still cloud platform landing, the server is a virtual technology in the computer to operate the foundation, the terminal can use a low configuration of the PC, so the laboratory hardware equipment will greatly increase the use of time The cost of management will be reduced accordingly.

Virtualized computing nodes and control nodes are an important part of server virtualization, and only when the equipment is relatively complete, can effectively promote the server virtualization, thereby enhancing its utilization and cost can be reduced. Which for the virtualized computing node, in the kernel virtual machine technology support, through the physical server to manipulate, so that a server can run on multiple virtual machines, virtual machine is also independent at this time, according to the actual needs Installation of the operating system. For virtualization control nodes, all components in the virtual environment can be viewed by the administrator, the management of resource pools, mirroring, servers, data storage and so on by a single CUI interface, in addition to the global real-time resources Detection, application load balancing and so on.

After the server virtualization, the data can be centralized management, the use of office PCs, mobile phones, etc. can be managed through the management platform for all servers and other effective management, business unfamiliar, routine maintenance can, and no longer rigidly adhere to the geographical location restrictions. When the physical server hardware damage, can not start the normal failure, the use of resources in the pool of the normal server can be restarted on the virtual machine, so that users can achieve the continuity of access. At the peak of the business visit, you can set up different virtual machines to effectively share the load, and its access to computing resources in accordance with their own priorities, so that in the case of high load business load balancing can be achieved. WINDOWS and so are the server virtualization after the virtual out of the operating system for research and other platforms, in addition to the support of the system template function, the server's experimental environment in just a few minutes can effectively build a successful, teacher teaching time can Effectively shortened. Virtual service has a backup interface, the use of storage comes with backup software, backup virtual machine, if the virtual machine operating system failure, the entire system and applications can be effectively restored. With the support of virtual technology, a comprehensive physical server based on a number of decentralized physical servers is built, and the cost of hardware purchases can be effectively reduced, and the average utilization of the server can be greatly improved. Management and maintenance will no longer need a longer time and more manpower, so the application of virtual technology to the computer laboratory construction management, the cost will be effectively reduced.

\section{Conclusion}

In this paper, based on the shortcomings of the traditional computer labs, this paper puts forward the virtual cloud technology to construct the virtual cloud computer experiment platform, and compares the traditional computer experiment teaching platform and the virtual cloud computing teaching experiment platform. Computer experimental resources, virtualization and the form of cloud for users unlimited time, place free access to use. It provides a more diverse, safe, stable and free experimental environment for experimental teaching and scientific research, which reduces the construction funds of the laboratory and improves the management and maintenance effect of the laboratory. Improve the laboratory utilization of experimental resources. Virtual cloud technology in the laboratory construction of the application prospects are very broad. 


\section{Acknowledgements}

This article is the research project of Xianyang Normal University 2015, 2017 scientific research project;

Project name: Research on the open management mode of open laboratory in colleges and universities, and the pragmatic empathy from the speech of Xi Jinping.

No .: 15XSYK005, XSYK17024;

\section{References}

[1] Wang Hong, Shi Yiping. University computer laboratory exclusive practice cloud system [J]. Fujian Computer. 2017 (08)

[2] Deng Jianfeng. On the strengthening of computer lab management in colleges and universities of several suggestions [J]. Talent. 2017 (19)

[3] Pan Yuhong. On the management of computer labs in colleges and universities [J]. Private Technology. 2016 (11)

[4] Shi Nuo. University computer laboratory management and maintenance [J]. Computer fans. 2017 (03)

[5] Wen Junyuan. University computer laboratory management and maintenance [J]. Science and Technology Economic Guide. 2017 (16)

[6] Chen Yan. College computer laboratory management and maintenance strategy analysis [J]. Private Technology. 2017 (02) 